\title{
QUELQUES PAIRES ÉTYMOLOGIQUES FRANÇAIS - SERBE DU CHAMP LEXICAL DES VÊTEMENTS
}

\author{
UDC 811.133.1'373.614:811.163.41'373.614
}

\section{Mihailo Popović}

Université de Belgrade, Faculté de philologie

\begin{abstract}
Résumé. Nous nous proposons dans cet article de suivre l'évolution phonétique et sémantique de quelques mots français et serbes ${ }^{l}$ appartenant au lexique des vetements et ayant les étymons communs. Ce sont les mots qui ont la même origine indo-européenne ou latine et les mots d'origine arabe ou turque. Ces mots ont trait aux matières textiles, à la technique de fabrication des vetements et aux objets vestimentaires. L'analyse des changements de la forme et du contenu montre qu'il y a quatre rapports entre les paires étymologiques. $1^{\circ} \mathrm{Il}$ existe une ressemblance de la forme et l'identité du sens. $2^{\circ}$ L'identité du sens est bien manifeste, mais les formes sont très éloignées l'une de l'autre. $3^{\circ} \mathrm{La}$ ressemblance de la forme est évidente, mais pas celle du sens. $4^{\circ} \mathrm{Ni}$ le sens ni la forme ne paraissent pas avoir de traits communs. Les différences de forme et de sens proviennent de l'évolution propre à chaque langue, mais aussi de l'influence des langues véhiculaires et de l'analogie.
\end{abstract}

Mots-clés : étymologie, forme, sens, indo-européen, latin, arabe

\section{INTRODUCTION}

Le lexique des vêtements est caractérisé par une grande instabilité qui se manifeste de deux manières. D'abord, les mots circulent, il y en a qui disparaissent et de nouveaux qui entrent en usage. Un locuteur français n'emploie pas les mots comme cotte, jaque, spencer, de même qu'un locuteur serbe n'emploie plus les mots du genre anterija, libada, koporan. D'autre part, il arrive que la forme reste, mais le contenu change. Ainsi les mots français jupe et robe ne désignent-ils pas aujourd'hui la même chose qu'auparavant, de même que le mot serbe haljina. Et à l'inverse, il arrive que la forme change et pas le

Submitted July 1, 2019; Accepted September 9, 2019

Corresponding author: Mihailo Popović

University of Belgrade, Faculty of Philology

E-mail: mihailopopovic@eunet.rs

${ }^{1}$ Les faits linguistiques dont on discute dans cet article sont relatifs à la langue que l'on parle en Serbie, en Croatie, en Bosnie-Herzégovine et au Monténégro et que, depuis l'éclatement de la Yougoslavie, l'on nomme le serbe, le croate, le bosniaque et le monténégrin. 
contenu. Autrefois on appelait tricot ce qu'on appelle aujourd'hui chandail ou pull, ainsi qu'on appelait en serbe čakšire ce qu'on appelle maintenant pantalone. Le lexique des vêtements est en grande partie sujet aux influences étrangères. Parmi une quinzaine de pièces de vêtement les plus usuelles, la moitié a des noms d'origine étrangère ${ }^{2}$ aussi bien en français qu'en serbe : blazer, costume, jupe, bluza, pantalon, pull, short, slip, veste ; bluza, čarape, džemper, jakna, kapa, kaput, kostim, majica, pantalone, sako...

Les mots français et serbes analysés dans cet article sont soit issus du même étymon indo-européen ou latin, soit empruntés à l'arabe et qui sont entrés dans la langue française en passant par différentes langues véhiculaires et dans la langue serbe par l'intermédiaire du turc. Nous avons suivi l'évolution de la forme et du contenu de quelques étymons communs au français at au serbe.

\section{MATÉRIAUX}

\subsection{Chanvre - konoplja}

Bien qu'à première vue on ne remarque aucune ressemblance de forme (les deux mots n'ont pas un seul phonème en commun), ces mots ont le même étymon : lat. cannabis,

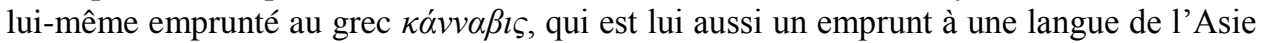
Mineure. Les deux mots présentent des irrégularités dans l'évolution de la forme.

Le mot latin a subi de nombreux changements lors de son évolution vers la forme française actuelle. Le [b] intervocalique faiblit en [v], ce qui est tout à fait régulier (habere $>$ avoir) ${ }^{3}$. Le mot latin étant un proparoxyton, le [a] de la pénultième cesse d'être prononcé et la voyelle dans la syllabe finale est, jusqu'au XVII ${ }^{\mathrm{e}}$ siècle, conservée sous la forme d'un [e]] central. Le premier [a], accentué, ne change pas, étant entravé. Plus tard, il subira la nasalisation au contact de la consonne nasale derrière lui. Le $[\mathrm{k}]$ se palatalise devant [a]. Ce qui reste inexpliqué, c'est le [R] final. En ancien français l'on rencontre les formes chanve et chanvre. Dans les dialectes français, l'on retrouve de nombreuses formes des deux types: chanve, chambe, chanebe, cambe, canebe; chambre, charbe, cambre, parmi d'autres (FEW, II, 210).

En serbe, au lieu des deux [a] du mot latin ou grec on a deux [o], comme d'ailleurs dans toutes les langues slaves : rus. конопля, slovène konoplja, pol. konopie, tchèque konopi, bulg. коно́n. On peut remarquer le même changement de [a]... [a] > [o]... [o] dans les toponymes d'origine latine Cattarum > Kotor, Massarum > Mosor (Skok, 2, 459).

Le [b] intervocalique est devenu [p] en latin vulgaire (*cannapis, canapus).

La syllabe finale $[\lambda \mathrm{a}]$ se rencontre aussi en slavon d'église, en russe et en ukrainien (конопля) et en slovène (konoplja). Skok suppose une forme * canapia en latin vulgaire, parallèle à la forme romane canapa ${ }^{4}$, hypothèse étayée par les formes ganiepa en istroroumain, cianaipe en frioulan (Skok, 2, 30), de même que konopie en polonais et konopje en sorabe (Vasmer : конопля). Le groupe [pj] change en [p $\lambda]$, comme dans kap $+j a>$ kaplja, snop + je > snoplje.

\footnotetext{
${ }^{2}$ C'est-à-dire, qui ne sont pas hérités, respectivement, du vieux fond lexical latin ou slave.

${ }^{3}$ Cannabis évolue en *annapis et canapus en latin vulgaire, ce qui ne change pas le résultat de l'évolution de la consonne bilabiale : [p], [b] > [v] ; ripa > rive, faba > fève.

${ }^{4}$ D'où l'hésitation sur le genre en français. Jusqu'au XVIII siècle, chanvre est aussi bien un nom masculin que féminin.
} 
Le chanvre et konoplja ont le même contenu, ils désignent tous les deux la plante industrielle canabis sativa.

Il existe en serbe un autre mot qui provient du même étymon et qui apparait sous deux formes : kanap et konop (ficelle, cordelette). La première est un emprunt à l'italien canapo (Skok, 2, 30), avec une influence hypothétique du mot turc kınnap (Škaljić 1985, 390). La deuxième est plus près des formes slaves et apparaît très souvent avec le suffixe -ac qui n'a aucun rôle sémantique : konop et konopac sont synonymes.

En français aussi il y a un autre mot issu de cannabis : canevas. Cette forme provient des dialectes du nord qui n'ont pas connu la palatalisation de [k] devant [a]. En ancien français l'on rencontre des formes chanevas et chenevas. Le serbe a emprunté ce mot au français sous la forme kanafas.

\subsection{Laine - vuna}

Les deux mots tirent leur origine de la racine indo-européenne wel- / wol-, élargie par le morphème -na. Les mots des différentes langues indo-européennes désignant la laine, issus de cet étymon, contiennent, en principe, trois consonnes : [w] ([v]), [1] et [n], par exemple, en tchèque vlna. En grec ancien et en latin, la consonne initiale, la spirante bilabiale [w], s'est amuïe : $\lambda \tilde{\eta} v o \varsigma$, lana $^{5}$. Dans les langues germaniques, on remarque l'absence de [n] : all. Wolle, angl. wool, néerl. wol. Les mots désignant la laine dans les langues slaves ont toutes les trois consonnes : rus. во́лна, bulg. вълна, slovène volna etc. La spirante bilabiale [w] est devenue fricative labio-dentale [v], ce qui est un changement régulier (*wid- > vid, ${ }^{*}$ wed- > voda, ${ }^{*}{ }^{*} \mathrm{wl}^{\mathrm{w}}>$ vuk).

L'évolution phonétique de ces deux mots est régulière. En français, on part de l'étymon latin lána. Le premier [a], accentué et libre, se diphtongue devant une consonne nasale en [ai], dont on a le souvenir dans l'orthographe. Cette diphtongue subit la nasalisation : [ãi]. Ensuite, le premier élément de la diphtongue, sous l'influence du second, se ferme : [ $\widetilde{\varepsilon} i]$. Plus tard, la diphtongue perd son deuxième élément pour devenir monophtongue nasale : [ $\tilde{\varepsilon}]$. À partir du $\mathrm{XVI}^{\mathrm{e}}$ siècle, cette voyelle se dénasalise et devient voyelle orale $[\varepsilon]$. Le deuxième [a] de l'étymon latin, étant inaccentué, faiblit en voyelle centrale [e] qui se labialisera en [œ], faiblira en [ə] au XVII ${ }^{\mathrm{e}}$ siècle pour disparaître bientôt après : laine [lkn].

L'étymon du mot serbe est le vieux slave вльна, qui perdra la voyelle réduite ${ }^{6} \mathrm{~b}$ : [vlna] avec un [1] syllabique. Dans cette position, [1] se vocalise en $[\mathrm{u}]^{7}$ : vuna, comme [vlk] > vuk, [dlg] > dug, [vlttci] > vući, etc. (Belić 1976, 76).

Il existait en vieux slave un homonyme вльна (vague) ${ }^{8}$, qui a été remplacé en serbe par le mot val, issu de la même racine indo-européenne wel- (rouler) ou par le mot talas, d'origine grecque.

Quelques autres mots français désignant les tissus proviennent de la même racine indoeuropéenne. Velours, par fausse régression de l'ancien français velous (< lat. villosus). Flanelle est un emprunt à l'anglais flannel, issu du gallois gwlan (laine) (Picoche 2009,

\footnotetext{
${ }^{5}$ Mais vellus de la même racine. Cf. serbe vlas.

${ }^{6}$ Nous utilisons la transcription traditionnelle $[ъ]$ et $[$ ь $]$ pour les deux voyelles réduites dont les différences se sont effacées très tôt, mais qui ont laissé des traces différentes dans les langues et les dialectes slaves.

${ }^{7}$ Cette vocalisation n'a pas eu lieu dans certains dialectes du sud-est de la Serbie. Cf. le toponyme Žlne, village près de Knjaževac.

${ }^{8} \mathrm{Cf}$. en russe волна́ (vague). Le mot habituel pour désigner la laine en russe est иерсть.
} 
300). Velvet est aussi un emprunt à l'anglais velvet, qui provient lui aussi du latin villosus

(FEW, XVIII : 129). Le serbe a emprunté ces trois mots : velur, flanel, velvet.

Il n'y a pas de différences sémantiques entre les contenus des mots laine et vuna.

\subsection{Coton - ćeten}

Le coton est cultivé en Inde depuis plusieurs millénaires. Les Arabes y ont connu le cotonnier et ont commencé très tôt à le cultiver en Égypte, en Espagne méridionale et en Sicile. Le mot coton provient de l'arabe quțun, par l'intermédiaire de l'italien (cotone), et non pas par l'espagnol comme beaucoup d'emprunts à l'arabe au Moyen-âge. L'espagnol a emprunté les substantifs arabes avec l'article arabe agglutiné et ces mots sont passés en français sous cette forme ${ }^{9}$. L'arabe quțun est emprunté sur la péninsule ibérique sous la forme algodón en espagnol et algodão en portugais.

Le mot italien s'adapte en français d'après les règles d'assimilation caractéristiques pour les emprunts anciens. La voyelle finale inaccentuée cesse d'être prononcée (comme dans cannone > canon, soldato > soldat). Le [o] devant la consonne nasale se nasalise : [kotõ].

Le même étymon arabe, sous la forme katān (Nişanyan: keten, Skok, 1, 356), se retrouve dans le mot turc keten. Curieusement, ce mot ne désigne pas le coton, mais une autre plante textile, le lin. Le serbe avait emprunté ce mot sous la forme ćeten. Lors de l'adaptation phonétique, il y a eu un seul changement : le [k] devant [e], déjà palatalisé en turc [kjeten], devient consonne affriquée alvéolo-palatale sourde [t6]. Ce type d'adaptation est tout à fait régulier : kebe > ćebe, kel >ćela, kebap > ćevap.

Ćeten désignant le lin figure dans le dictionnaire de Vuk (1851), mais il n'a pas résisté à la concurrence du mot autochtone lan et il est aujourd'hui complètement hors d'usage.

On le retrouve dans le mot composé ćeten-alva, confiserie faite à la base de tahini ou de semoule et de sucre. Lors de sa confection, la pâte prend la forme de fines fibres qui ressemblent à celles du lin. Ce composé est entièrement emprunté au turc keten halva (keten helva en turc contemporain).

\subsection{Lin - lan}

La racine de ces deux mots est $l i$-no ${ }^{10}$ (Pokorny 1959, 691). Cette racine se retrouve dans toutes les langues européennes, soit par des emprunts très anciens, soit par le développement indépendant : gr. גivov, lat. lìnum, vx. sl. *lınъ, rus. лён, all. Lein, angl. linen.

L'étymon du mot français est le latin lìnum. Le [ī] long latin ne change pas, le [m] final cesse d'être pronocé au $\mathrm{I}^{\mathrm{er}} \mathrm{s}$. av. n. è. et le [u] final, inaccentué s'ouvre au $\mathrm{V}^{\mathrm{e}} \mathrm{s}$. ([lino]), pour disparaître au VII ${ }^{\mathrm{e}}$ : [lin]. Au XIII ${ }^{\mathrm{e}}$ s., le [i] se nasalise devant la nasale : [iinn]. Un siècle plus tard, [ĩ] nasal s'ouvre d'un degré : [lẽn] et continue de s'ouvrir : [lẽn]. Au cours du XVI et du XVII ${ }^{e}$ s., lors de la dénasalisation en syllabe ouverte, la consonne nasale cesse d'être prononcée : [lĩ], lin.

Le mot serbe lan s'est développé de la racine vieux slave *lbnъ (Skok, 2, 266). Cette racine contient deux voyelles réduites. Dans la position forte, elles se transforment en d'autres voyelles et dans la position faible, elles disparaissent. La première, [b] qui provient du [1̄] indo-européen, accentuée, et de ce fait dans la position forte, devient [a]

\footnotetext{
${ }^{9}$ Par exemple : alcôve, abricot, azimut.

${ }^{10}$ Bien que le lin provienne de l'Asie, cette racine ne se trouve que dans les langues de l'Europe (Skok, $2: 266$ ).
} 
en serbe, en slovène (lan) et en slovaque (l'an), [о] en russe (лён $)^{11}$ et [е] dans les autres langues slaves (bulg.: лен, macéd.: лен, tchèque : len, pol. : len). À la fin du mot, la voyelle réduite est dans la position faible et elle s'amuit.

Lin et lan ont le même sens et désignent la plante et, par métonymie, le tissu qu'on fait de cette plante.

L'aspect rectiligne des tiges de lin est la base sémantique des dérivés latins linea et linearis. Le champ étymologique de lin en français est composé de mots ligne (avec de nombreux dérivés), linceul, linge, linotte, linoléum. En serbe, à part les emprunts (linija, linearan, lenjir, lincun, etc.), lan a quelques dérivés: lanar (linier), lanara (usine de lin), lanen (de lin), lanište (linière).

\section{TECHNIQUES}

\subsection{Coudre - šiti}

Rien dans la forme de ces deux verbes n'indique leur parenté. Et pourtant, ils proviennent de la même racine indo-européene : sìn- / siü - (Pokorny 1959, 915). Cette racine se trouve dans les mots du champs sémantique de la couture dans les langues indoeuropéennes : sīvyati (sanscrit), suere (latin), siujan (gothique), to saw (anglais), siūti (lituanien), uumb (russe).

Le verbe français s'est développé de la forme préfixée de suere: consuere (littéralement, coudre ensemble, forme analogue à zašiti en serbe). Le latin classique consúere subit trois changements en latin vulgaire. Le [n] cesse d'être prononcé devant [s] (comme dans mensa > mesa, insula > isula). Ensuite, le [u] en hiatus se ferme d'un degré, ce qui entraîne le déplacement de l'accent vers le début du mot : [kóswĕre] et le [w] disparaît : cósěre (comme dans battúere $>$ báttěre $>$ battre). Le [s] entre deux voyelles se sonorise : [kózere], le [e] inaccentué s'amuit: [kózrę], entre [z] et [r] s'intercale la consonne épenthétique [d]: [kózdrę], le [z] implosif disparaît: [kódrę], le [o] initial se ferme en [u] et la voyelle finale, antérieurement affaiblie en [e] central disparait: [kudR] coudre ${ }^{12}$. Les mots couture, suture, accoutrer appartiennent au même champ étymologique.

Dans les langues slaves, le groupe [sj] de l'indo-européen $s i-s i \bar{u}$ - devient [S] $]^{13}$ (Ivšić 1970, 149): rus. uить, bulg. ши́я, macéd. uиe, slovène, tchèque šiti, slovaque šit', pol. szyć. Donc, le verbe est formé de la base ši- et du suffixe de l'infinitif, dans les langues qui en ont un. Les mots šilo, šiljak, šav, švalja font partie du même champ étymologique.

Coudre et šiti désignent la même technique de la confection des vêtements.

\subsection{Plier - plesti}

Les deux verbes proviennent de la racine pel- / plek-. Cette racine a donné de nombreux lexèmes dans les langues indo-européennes : gr. $\pi \lambda \varepsilon ́ \kappa \omega$, lat. plicare, plectere, avec un très grand nombre de dérivés (par exemple, simplus, duplus, complexus, applicare, complicare, explicare, supplicare etc.), angl. to fold, all. falten.

\footnotetext{
${ }^{11}$ La présence ancienne de la voyelle réduite a eu pour conséquence la palatalisation de la consonne précédente en slovaque et en russe.

${ }^{12}$ M. Popović, 2014: 40, 110

${ }^{13} \mathrm{Cf}$. nositi - nošen, tesati - tešem. Une phase transitoire entre [sj] et [S] se retrouve dans la prononciation du mot sjutra dans certains parlers au Monténégro, orthographié aussi śutra (il s'agit, en fait, d'une variante combinatoire).
} 
On remarque la ressemblance formelle entre plier et plesti dans le groupe consonantique initial [pl]. Le français, à la différence des autres langues romanes, a conservé le groupe initial [pl], de même que le serbe (et les autres langues slaves) : plak- / plag- > plaindre, plakati ; pleu- > pleuvoir, ploviti (Pokorny 1959, 832, 837).

Deux verbes français sont issus de l'étymon latin plicare : ployer et plier. Ployer est le résultat de l'évolution régulière. Le [1] court se ferme en latin vulgaire : [plekare]. Le [k] intervocalique faiblit progressivement pour devenir [j] : [plejare]. Ce [j] provoque la diphtongaison de [a] accentué en [ie]. Le [j] se vocalise ensuite et forme la diphtongue [ei] avec la voyelle précédente, de sorte qu'on trouve deux diphtongues successives : [pleiíer]. Entre-temps, le [e] final posttonique a cessé d'être prononcé. La diphtongue [ei] devient [oi] par la différenciation du point d'articulation: [ploiíer]. Ensuite, il se passe une réduction de la différence d'aperture de la première diphtongue [oi] > [ue]. Dans la deuxième, l'accent se déplace sur le deuxième élément [plueiér] ce qui provoque la fermeture du premier: [pluejér]. La diphtongue [ue] évolue en [wa] et le [r] cesse d'être prononcé en position finale: [plwaje]. La forme plier a été refaite à partir du XIII ${ }^{\mathrm{e}}$ siècle sur le singulier du présent (je pli) ${ }^{14}$.

La base verbale dans les langues slaves est plekt- ${ }^{15}$. Même avant l'époque protoslave, le groupe [kt] se réduit en [t] (Ivšić 1970, 146 ; Nikolić 1978, 101), et on a une base plet-. Avec le sufixe de l'infinitif, on obtient [pletti]. Entre deux [t] se dévelope la consonne épenthétique [s]: [pletsti] (Belić 1976, 135 ; Ivšić 1970, 128). Le premier [t], implosif, disparaît: plesti. Les mots serbes plot, splet, spletka, pleter appartiennent au même champ étymologique.

Le lien sémantique entre plier et plesti n'est pas très fort. C'est l'étymon latin plectere (tresser, entrelacer) qui est plus proche du verbe serbe. De très nombreux mots français sont issus de cet étymon, mais aucun ne désigne la technique de production d'objets en laine. L'équivalent français de plesti est le verbe tricoter ${ }^{16}$.

Deux mots d'emprunt du domaine de la couture en serbe proviennent de l'étymon pel/ plek- : plisirati (< fr. plisser) et falta (pli, < all. Falte).

\section{OBJETS}

\subsection{Chapeau - kapa}

Les deux mots sont issus du bas latin cappa, qui pourrait être apparenté à caput. Cappa désignait le capuchon et, par métonymie, le manteau à capuchon. Deux mots français proviennent directement de cet étymon: chape et cape, ce dernier par l'intermédiaire du provençal. L'étymon direct de chapeau est le bas latin cappellus, diminutif de cappa. Le [k] initial subit la palatalisation devant [a]. Le [a] ne change pas, étant entravé. La géminée [pp], avant de se simplifier, empêche le changement de [p] en [v] (à la différence de sapere > savoir). La voyelle dans la syllabe finale disparaît: [tgapels]. Le [1] implosif se vocalise en formant une triphtongue avec un [a] de transition: [tgapeaus]. L'affriquée initiale perd l'élément dental et devient [J], le [s] final cesse d'être prononcé, et la triphtongue se réduit

\footnotetext{
${ }^{14}$ L'orthographe de l'époque. La raison de ce changement est évidente pour le verbe nier, parce qu'il fallait éliminer l'homonymie de noiier qui pouvait provenir de necare aussi bien que de negare. Plicare a produit les doublets, tandis que la forme proier a été remplacée par prier.

${ }^{15}$ Comme en lat. plectere, gr. $\pi \lambda \varepsilon \kappa \tau \eta \dot{~(f i l e t), ~ e t ~ d o n t ~ l e ~ r e f l e t ~ s e ~ t r o u v e ~ e n ~ a l l e m e n d ~ f l e c h t e n ~(t r e s s e r) . ~}$

${ }^{16}$ De la même origine que le verbe serbe štrikati (< all. stricken), synonyme de plesti (FEW, $17: 256$ - 258).
} 
graduellement en [o]: [ $\left.\int a p o\right]$. Cette forme résulte donc soit du cas sujet du singulier, soit d'après le cas régime pluriel (de cappellos). Le cas régime singulier en ancien français était chapel (de cappellum).

Le champ étymologique de cappa en français est large: chaperon,chapelet, chapelle, chapelain; échapper, rescapé, escapade; capot, décapotable; capucin, capuchon; képi...

Le mot kapa est venu dans la langue serbe par l'intermédiaire de l'italien cappa. Il n'a subi aucun changement de forme et il est tel qu'il était en bas latin. On le trouve sous cette forme aussi en slovène et en macédonien.

Il est intéressant de remarquer le mouvement métonymique du haut en bas et l'inverse du mot latin cappa et des mots qui en sont issus. Cappa désigne d'abord le capuchon, et ensuite le manteau à capuchon. Les mots français chape et cape, ainsi que l'italien cappa désignent une sorte de manteau avec ou sans capuchon. Le dérivé bas latin cappellus dénomme une coiffure, de même que le serbe kapa.

Les mots chapeau et kapa, tout en désignant un vêtement qui couvre la tête, n'ont pas exactement le même sens. Kapa a un sens plus large, c'est une coiffure quelconque qui peut être un bonnet, une casquette, un calot etc, à l'exception de chapeau. Le trait distinctif entre les deux mots et „le bord“. L'équivalent de chapeau en serbe est šešir.

Le champ étymologique en serbe contient encore quelques mots. $\breve{S} a p k a$, que l'on trouve dans toutes les langues slaves, en roumain, en hongrois et en turc, sous différentes formes, est un emprunt probablement au russe шапка. Vasmer suppose pour les mots slaves un emprunt du moyen haut allemand schapël, lui-même emprunté à l'ancien français chapel (Vasmer : шапка). En serbe, le trait distinctif est la visière et šapka désigne une casquette faisant partie de l'uniforme militaire ou civile. Le mot français chapska est un emprunt au polonais czapka ${ }^{17}$.

Kaput est un pardessus d'hiver. Klajn et Šipka $(2006,581)$ considèrent que le mot est emprunté à l'italien cappotto, tandis que Skok suppose une influence du turc kaput (Skok, 2, 39), lui-même emprunté au français capote (Nişanyan: kaput). L'hypothèse la plus probable est qu'il s'agit de l'emprunt du mot allemand Kaput ${ }^{18}$, aujourd'hui tombé en désuétude. Vuk note l'existence de la forme kapot au Monténégro (p. 273), aujourd'hui désuète ${ }^{19}$.

Kapuljača, „capuchon“, dérivé de kapa, avec le suffixe -ača (var. -uljača) sans contenu sémantique précis.

\subsection{Gilet - jelek}

Le mot français est un emprunt à l'espagnol jileco ${ }^{20}$ (Picoche 2009, 258). Le mot apparaît en français vers le milieu du XVI ${ }^{\mathrm{e}}$ siècle, à l'époque où la lettre espagnole « $\mathrm{j}$ » pouvait encore être prononcée [3]. Quant à la graphie de l'initiale elle pourrait être influencée par la forme gileco. Le changement de la syllabe finale en français serait dû à l'influence des mots comme corset, mantelet (FEW, 19:200, TLF). Le mot espagnol est un emprunt à l'arabe d'Algérie jaleco ${ }^{21}$, lui-même emprunté au turc yelek. Ce mot se

\footnotetext{
${ }^{17} T L F$. Cf. «C'était une de ces coiffures d'ordre composite, où l'on retrouve les éléments du bonnet à poil, du chapska, du chapeau rond, de la casquette de loutre et du bonnet de coton... » (Flaubert, Madame Bovary, TLF).

18 "Kaput, kaputrock auch kapot, m. - mantel mit einer kappe, cucullus, von it. cappotto, franz. capot." (DWB).

${ }^{19}$ V. aussi $R M S$ : kapot.

${ }^{20}$ Il existe des variantes gileco, jaleco et, en espagnol contemporain chaleco.

${ }^{21} F E W$ et $T L F$ citent comme source l'arabe maghrébin ğalīka (< turc yelek), sorte de camisole sans manches portée par les esclaves chrétiens sur les galères.
} 
trouve dans les langues et les parlers romans de la Méditerranée : sicilien gileccu, cileccu, sarde gilèccu, génois gilecco, corse ghjileccu, portugais jaleco. (FEW, $19: 200)$.

Le mot serbe est identique à son modèle turc : jelek. Ce mot se rencontre dans les langues des pays qui avaient fait partie de l'empire ottoman : gr. үєһغ́ко, macéd. елек, bulg. еле́к, alb. jelek, roum. ilic.

Gilet et jelek désignent à peu près la même pièce de vêtement, une sorte de veste courte et sans manches ${ }^{22}$. Et pourtant ces deux mots ne fonctionnent pas de la même manière dans leurs langues respectives. Gilet est un lexème couramment utilisé et qui connaît une expansion (gilet de sécurité, gilet de sauvetage, gilet pare-balles). Jelek aujourd'hui désigne une pièce du costume folklorique et s'emploie très rarement dans la langue courante. À sa place on emploie prsluk, dérivé de prsa (poitrine). Pour désigner l'élément d'un costume trois pièces, le serbe a emprunté le mot français, žile. Ainsi le serbe possède-t-il les doublets jelek et žile ${ }^{23}$.

\subsection{Jupe - džube}

Les deux mots proviennent de l'arabe ğubba, veste de dessous. Ce mot est entré en ancien français au XII ${ }^{\mathrm{e}}$ siècle par l'intermédiaire de l'ancien italien du sud jupa, veste d'homme ou de femme d'origine orientale (TLF, d'après $F E W)$. En passant en ancien italien, le mot arabe a subi une modification : la géminée «bb» s'est simplifiée et s'est désonorisée. En ancien français, l'affriquée [d] italienne $(<\breve{\mathrm{g}}$ arabe $)$ perd l'élément dental est devient $[3]^{24}$. Le $[\mathrm{u}]$ est prononcé $[\mathrm{y}]$ et la voyelle finale inaccentuée faiblit progressivement pour s'amuïr beaucoup plus tard : [3yp].

Le mot arabe s'est répandu dans les langues de la Méditerranée : it. giubba, esp. juba et avec l'article arabe aljuba, catalan jupa, port. aljuba. Le [u] du mot arabe est parfois prononcé [i] dans les dialectes italiens : giba, gipa. Il en est de même dans les dialectes français: provençal gipa, bourguignon jipe, champenois gipe (FEW, 19: 57 - 58). L'allemand a emprunté le mot aux langues romanes sous les formes Joppe (var. Jope, Juppe, Jüpe) Schaube, Schube ( $D W B$ : Jacke). De là, le mot est passé en polonais (jupa, région.), en ukrainien (юna) et en russe (юбка).

Les mots issus de l'arabe ğubba se sont propagés dans la péninsule balkanique de deux sources $^{25}$. De l'allemand: jopa, jopica, en slovène, jupa, en Croatie et $\check{s} u b a^{26}$ dans certaines régions de la Croatie et de la Serbie (Skok, 3, 420). Du turc cübbe (< ar. ğubba) : serbe džube / džuba, macéd. иубe, bulg. джубé, alb. xhybe, rum. giubea.

En passant dans la langue serbe le mot turc subit une seule modification. Le [y], qui n'existe pas dans le système phonologique serbe, est assimilé comme [u], ce qui est une adaptation régulière des mots turcs contenant cette voyelle ${ }^{27}$. Il existe en serbe une variante $d \check{u} u b a$.

\footnotetext{
${ }^{22}$ Par extension, gilet peut désigner un tricot à manches longues qui se boutonne par devant (TLF).

${ }^{23}$ D'autres exemples de doublets dont un élément est venu par le turc et l'autre par une langue européenne : ćošak-kiosk, šerbet - sirup, ajvar-kavijar, marama-macramé.

${ }^{24}$ Le mot est attesté en ancien français vers la fin du XII , au moment où le changement [d] $>$ [3] était en train de se produire.

${ }^{25}$ Skok signale l'influence italienne, par l'intermédiaire du vénitien dans džupa, noté par Vuk au Monténégro et en Herzégovine (Skok, $3: 420, V u k: 861$ ).

${ }^{26}$ Avec le trait sémantique «fourrure », de même qu'en russe $и у б a$. Šubara est dérivé de šuba.

${ }^{27}$ Par exemple : dügme $>$ dugme, dükkan $>$ dućan, küp $>c$ ćp, gübre $>$ đubre.
} 
Tous ces mots, répliques de $\breve{g} u b b a$ dans les différentes langues, désignent un vêtement d'homme ou de femme que l'on porte au-dessus des autres vêtements, une sorte de manteau ou de veste.

En français, jupe avait d'abord désigné un vêtement d'homme ou de femme couvrant le buste jusqu'au dessous de la ceinture. Au début du XVII ${ }^{\mathrm{e}}$ siècle, ce mot commence à désigner le vêtement féminin qui va de la taille vers les pieds et qui est plus ou moins long selon la mode. Il a remplacé le mot cotillon. Au pluriel, il désignait l'ensemble formé par la jupe et les jupons et qui est la base sémantique de l'expression être dans les jupes de sa mère. Le serbe a emprunté le dérivé jupon au français : žipon.

En serbe, le mot $d z ̌ u b e$ a deux acceptions : 1. Tunique longue (jubba), généralement de couleur noire, portée par les religieux musulmans. 2. Veste d'homme ou de femme.

Tandis que le mot jupe est bien vivant en français, džube est vieilli et d'un emploi très rare ou régional en serbe contemporain. Il a été suppléé par jakna.

\subsection{Jaque - jakna}

Jaque désignait un vêtement à manches qui couvrait le torse jusqu'au-dessous de la ceinture et que l'on portait au Moyen Âge. Par ellipse, jaque [de mailles] désignait la cotte de mailles de soldats de l'Antiquité et du Moyen Âge. L'étymologie de ce mot n'est pas établie avec certitude. Il proviendrait du sobriquet jacques donné autrefois aux paysans par les nobles et les bourgeois, d'après le prénom Jacques, parce que ce vêtement court et simple ressemblait à celui qu'ils portaient. L'hypothèse d'après laquelle ce mot proviendrait de l'arabe šakk (cotte de mailles), par l'intermédiaire du catalan ou de l'espagnol, parait peu vraisemblable du point de vue phonétique et du point de vue historique. En effet, c'est le mot français qui est passé dans les autres langues romanes $(F E W, 5: 11)$.

Le mot s'est propagé dans toutes les directions, avec un sens général de veste : esp. jaco, cat. jac, port. jaqué, it. giacca, all. Jacke, suéd. jacka, norv. jakke. Dans certaines de ces langues le mot est usuel, dans d'autres archaïque.

Le mot serbe jakna est un emprunt à l'allemand Jacke, lui-même emprunté au français jaque au $\mathrm{XV}^{\mathrm{e}}$ siècle $(D W B)$. Le mot français avait dû passer en allemand par voie écrite, ce qui explique la prononciation de la consonne initiale [j]. Les emprunts postérieurs sont plus proches de la prononciation française (Jackett, Jalousie, Jargon, Journal). La voyelle finale du mot français, qui à l'époque de l'emprunt était prononcée [œ], est transmise comme [e].

Pour garder le genre féminin en passant en serbe, le mot a pris une terminaison en [a]. Et il a une consonne de plus que son modèle allemand, de sorte qu'il se termine par un faux suffuxe -na.

Si dans le cas de l'emprunt du mot écrit la forme est susceptible de changer à cause d'une mauvaise lecture de la langue donneuse (par exemple, fr. jaque [zakœ] ${ }^{28}>$ all. Jacke [jake]), dans le cas de l'emprunt oral, le mot peut changer de forme à cause d'une mauvaise audition ou une mauvaise interprétation. C'est ainsi que de nombreux substantifs empruntés à l'allemand se terminent en -na en serbe ${ }^{29}$. Les raisons de ce changement de forme sont à la fois morphologiques et phonétiques. D'abord, ce n'est pas la forme du singulier des noms

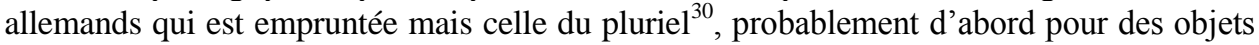

\footnotetext{
${ }^{28}$ La prononciation au moment de l'emprunt.

${ }^{29}$ Surtout dans le vocabulaire technique qui est en grande partie d'origine allemande et qui est généralement transmis par la voie orale : buksna (< Buchse), dizna (<Düse), hilzna (< Hülse), klocna < (Klotz), šelna (< Schelle) etc.

${ }^{30}$ Comme quelques emprunts en français : spaghetti / spaghettis, macaroni / macaronis, ravioli / raviolis.
} 
qui vont par deux (sokna < Socke / Socken, manžetna < Manschette /Manschetten < fr. manchette, kamašna < Gamasche / Gamaschen < fr. gamache) ou pour ceux que l'on conçoit souvent au pluriel, soit à cause de leur petite taille (nitna $<$ Niete / Nieten, šajbna $<$ Scheibe / Scheiben), soit parce qu'ils sont généralement employés par plusieurs (felna < Felge / Felgen, dizna < Düse / Düsen). Ce genre d'adaptation s'est ensuite propagé à beaucoup de substantifs féminins (rerna $<$ Röhre, mašna $<$ Masche, tašna $<$ Tasche, rolna $<$ Rolle, etc). D'autre part, le pluriel des mots allemands en -en était perçu comme se terminant par un groupe de consonnes : Jacken *[jakn] au lieu de [jakən], ce qui était contraire aux lois de la distribution des phonèmes en serbe ${ }^{31}$. Avec le $-a$ du féminin le mot ne se termine pas par un groupe de consonnes: jakna.

À la différence des paires gilet - jelek et jupe - džube, c'est le mot français qui n'a qu'un emploi historique, tandis que le mot serbe est d'un emploi courant. Jakna désigne une veste qui descend jusqu'à la taille ou qui couvre les hanches.

Le serbe a emprunté directement du français le diminutif de jaque, jaquette : žaket.

\subsection{Maillot - majica}

La forme et le sens des deux mots présentent des points communs. Ces deux mots tirent leur origine du latin macula, qui avait deux acceptions. D'abord, ce mot signifiait « tache $»^{32}$ et « tacheture » de certains animaux et ensuite, par métaphore, « maille de filet ».

En français, le mot a eu une évolution phonétique régulière. Étant proparoxyton, le mot perd très tôt la voyelle en pénultième : [makla]. Le groupe [kl] se palatalise et devient $[\lambda]$ :

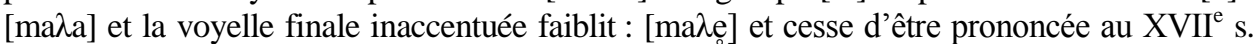
La consonne latérale $[\lambda]$ faiblit en perdant le contact prédorso-vélaire et aboutit à $[\mathrm{j}]$ : [maj], maille. Ce processus du relâchement de $[\lambda]$ avait duré du XIII ${ }^{\mathrm{e}}$ jusqu'à la fin du $\mathrm{XIX}^{\mathrm{e}}$ siècle $^{33}$.

L'évolution de macula était similaire dans d'autres langues romanes: it. maglia, esp. malla, port. malha.

En français, le sens de «tache » ne s'est conservé que dans quelques emplois spéciaux et a été évincé par le sens de «boucle de fil servant à faire un tissu, un treillis, un grillage » (Rey : 2096).

Maillot est issu de maille et, du point de vue historique, son premier sens est « lange dont on enveloppe un nouveau-né ». Il existe deux hypothèses sur l'étymologie du mot maillot. D'après la première, qui paraît plus vraisemblable, maillot provient, par changement de suffixe, de l'ancien et moyen français maillol, mailloel («lange »), dérivé de maille, par une analogie entre les bandes lacées du maillot et les bandes entrelacés. D'après la deuxième hypothèse, maillot est dérivé de l'ancien verbe mailloler « mettre au maillot », lui aussi issu de maille (Rey : 2097).

Le mot serbe majica est le diminutif de maja, mot emprunté au dialecte italien de Trieste $(\text { maia })^{34}$. Dans le dialecte triestin s'est produit le même changement qu'en français : le $[\lambda]$ de maglia (< lat. macula) a faibli et s'est transformé en [j]. Le même phénomène est observé dans certains dialectes croates de la côte adriatique (jubav, poje pour ljubav, polje). Maja est

\footnotetext{
${ }^{31}$ Autrefois, les seuls groupes de consonnes à la fin des mots étaient [st], [ft], [zd] et [3d]. Avec l'afflux de mots d'emprunt d'autres groupes de consonnes se sont trouvés à la fin des mots.

${ }^{32}$ D'où les mots savants en français : macule, maculer, immaculé.

${ }^{33} \mathrm{C}$ 'est pourquoi certains mots d'emprunts français en serbe se prononcent aujourd'hui avec $[\lambda]$ (detalj, paviljon, feljton, etc.).

${ }^{34}$ E. Kosovitz, Dizionario -Vocabolario del dialetto triestino e della lingua italiana, Trieste, 1890. (https://it.wikisource.org/wiki/Dizionario_-_Vocabolario_del_dialetto_triestino/M\#maia (4 / 4 / 2019)
} 
aujourd'hui senti comme vieilli et sa place est prise par majica qui a perdu la connotation diminutive.

Ces deux mots n'ont pas exactement le même sens à cause de la polysémie.

Maillot a trois sémèmes. D'abord, il a gardé son sens d'origine et désigne le lange dans lequel on enveloppe un bébé. Ce n'est qu'une acception historique, depuis qu'on a abandonné la pratique d'emmailloter les bébés. Ce sens est à la base de l'expression être au maillot, «être dans la première enfance ». Ensuite, maillot a, par analogie, commencé à désigner au début du $\mathrm{XIX}^{\mathrm{e}}$ siècle le vêtement souple qui moule le corps des danseuses et des danseurs au théâtre ${ }^{35}$. Et enfin, dans l'emploi courant, maillot désigne le vêtement souple et collant qui couvre le haut du corps et qui peut être un sous-vêtement (maillot de corps) ou un vêtement que l'on porte pour pratiquer un sport. Et, par l'ellipse, maillot désigne le maillot de bain et a remplacé le costume de bain. Le sème dominant de tous ces sens est « envelopper étroitement».

Le mot serbe majica désigne le vêtement léger et souple qui couvre le haut du corps. Il correspond au troisième sémème du mot français, mais pas toujours. Il pourrait être traduit en français, selon le contexte, par maillot [de corps], débardeur, tee-shirt ou polo.

\subsection{Robe - roba $^{36}$}

La similitude de forme est évidente, tandis que celle de sens n'est pas perçue en français et en serbe d'aujourd'hui. Les deux mots dérivent du germanique *rauba, «butin », « rapine »,

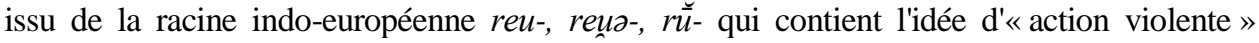
(couper, arracher, défoncer, s'emparer...). De très nombreux mots indo-européens proviennent de cette racine: gr. $\varepsilon \rho v \omega$; lat. rapere, ruere, rumpere, rapina, ruina ; all. rauben, Raub, rupfen, raufen; angl. rob, rape, reave, rip, rubble, serbe robiti ${ }^{37}$, slovène rop, ropati.

En français, le mot germanique *rauba a une évolution phonétique régulière. La diphtongue [au] se réduit à [o], au $\mathrm{V}^{\mathrm{e}}$ s., de même que dans les mots latins : [roba]. AuVII ${ }^{\mathrm{e}}$, le [a] final faiblit en [e] central: [robe]. À partir du XV siècle, la voyelle finale faiblit progressivement pour s'amuïr au XVII : [Rob] robe.

Le sens de «butin » est conservé en français jusqu'au XVI ${ }^{\mathrm{e}}$ siècle. Le dénominatif rober a été remplacé par voler, mais la forme préfixée, dérober, a subsisté, ainsi que l'expression à la dérobée. En ancien et en moyen français, le sémème de robe avait perdu le sème de « rapine » et le mot avait désigné « les biens mobiliers qui sont à l'usage d'une personne » ainsi que «la marchandise » $(F E W, 16: 674)$. Le glissement vers le sens actuel s'est fait probablement par la phase «vêtements en tant que butin», parce que les vêtements généralement faisaient partie du butin ${ }^{38}$. Le mot avait d'abord désigné l'ensemble de vêtements ${ }^{39}$. Cette acception n'a subsisté que dans le mot composé garde-robe. De là, le sens s'est restreint ensuite en «pièce de vêtement long» portée par les hommes et les

\footnotetext{
${ }^{35}$ Il est possible qu'il y ait eu une influence du nom propre Maillot, qui avait été bonnetier fournisseur de l'Opéra de Paris et qui aurait inventé ce costume d'après l'acteur F.-J. Talma (FEW, 6/ 1:55).

${ }^{36}$ Une analyse moins détaillée de cette étymologie a été publiée dans M. Popović, «Surprenantes étymologies communes au français et au serbe », Zbornik radova sa II međunarodnog naučnog skupa DEAF 2 (Dire, écrire, agir en français), Kragujevac, 2013, pp. 317 - 325.

37 « Piller, dévaliser ». Il existe un homonyme robiti, « réduire en esclavage », dérivé de rob.

${ }^{38} \mathrm{Cf}$. « Si vindrent a une bone vile qui la Filee avoit nom, et la pristrent ; et firent grant gaieng de proies, de prisons, de robes, de viandes », Villehardouin, La Conquête de Constantinople, 226. (Ils parvinrent à une bonne ville qui avait pour nom Philée ; ils la prirent et firent un grand butin de bétail, de prisonniers, de vêtements et de vivres.)

${ }^{39} \mathrm{Cf}$. «Mes n'an set plus, que nuz se trueve. Devant lui voit la robe nueve [...]. » Chr. de Troyes, Le chevalier au lion, 3024. (Tout ce qu'il sait, c'est qu'il se voit nu. Il aperçut devant lui des vêtements neufs [...].)
} 
femmes. Ce sens est conservé dans le syntagme robe de chambre. La robe est aussi un signe d'appartenance à un état ou une profession (gens de robe, noblesse de robe). Le sens actuel, issu d'une nouvelle restriction, apparaît au XII ${ }^{\mathrm{e}}$ siècle.

Le mot serbe est un emprunt à l'italien roba (< lat. méd. rauba < germ. *rauba). Lors du transfert, le mot n'a subi aucun changement.

Le mot italien roba a une polysémie développée. Il désigne des choses en général (au point de devenir un pronom indéfini), des biens de différentes sortes, des vêtements, des marchandises. Ce sont les deux dernières acceptions qui ont été transférées en serbe.

Le sens de « vêtements » a été courant au $\mathrm{XIX}^{\mathrm{e}}$ et dans la première moitié du $\mathrm{XX}^{\mathrm{e}}$ siècle ("Oficirska roba na njemu, zvijezda na kapi, a revolver u ruci") ${ }^{40}$, mais aujourd'hui il a vieilli. Roba «marchandise » est un mot tout à fait courant.

Il existe une variante ruba, signifiant uniquement «vêtements », aujourd'hui abandonnée $^{41}$. Il est possible qu'il y ait eu un croisement entre roba et la base slave $r u b$-, par proximité semantique avec des mots comme rublje, «linge », rubac, «mouchoir», rubina, « chemise » (Skok, $3: 151,163)$.

Il est intéressant de remarquer que robe / roba dans les deux langues autrefois pouvait désigner la femme en tant qu'objet sexuel : «Bonne robe - se dit d'une femme appétissante et ardente au plaisir » (Godefroy, 7: 209). Cette locution était encore usité au XVII ${ }^{\mathrm{e}}$ siècle : «Elle était fille à bien armer un lit... Ce qu'on appelle en français bonne robe ${ }^{42}$. Femme comme butin, bien que l'on possède ou marchandise ? C'est un exemple flagrant de la réification de la femme. Il en était de même en serbe : ,- Da mi je jednom povaliti na zemlju gologlavu varošku gospojicu, onu šišku u svilenim haljinama, ne bih žalio odmah umrijeti! [...] - Uf, više bih ja volio zdupiti jednu Turkinju šarenijeh gaća, neg da ti meni dadeš pola Mrčaja! [...] - Ima kod partizana i jedne i druge robe, dobro su oni to smislili.““43

\section{CONCLUSION}

Les mots français et serbes analysés qui ont le même étymon sont soit issus des racines indo-européennes (wel- / wol- : laine - vuna, siū- / siu - : coudre - šiti, pel- / plek-: plier plesti, reu- / reù- / rū- : robe - roba), ou latines (cannabis : chanvre - konoplja, cappa : chapeau - kapa, macula : maillot-majica), soit des emprunts très anciens à l'arabe ou au turc (quțun : coton-ćeten, ğubba : jupe-džube, yelek : gilet-jelek). Ces mots ont évolué du point de vue de la forme suivant les règles propres à chaque langue, mais aussi sous l'influence des langues véhiculaires et de l'analogie. Dans certains cas, les mots français et serbes ont le même sens (chanvre -konoplja, laine - vuna, lin - lan, coudre - šiti). Dans d'autres, les paires étymologiques appartiennent au même champ lexical et les différences sont dues à la polysémie ou au caractère archaïque de certains lexèmes (chapeau-kapa, gilet - jelek, jupe džube, jaque - jakna, maillot - majica). Dans quelques cas, on ne voit pas de trait sémantique commun entre les lexèmes (coton - ćeten, plier - plesti, robe - roba). La comparaison des formes et des contenus des paires étymologiques analysés permet d'établir quatre types de rapports. $1^{\circ} \mathrm{Il}$ existe une ressemblance de la forme et du sens (lin - lan, maillot-majica). $2^{\circ}$ Les formes sont différentes, tandis que le sens et identique (chanvre-konoplja, coudre - šiti).

\footnotetext{
${ }^{40}$ B. Ćopić, $R M S$.

41 „Oblači stajaću rubu radnim danom“, L. Lazarević, „Na bunaru“, Pripovetke, Sarajevo, 1989, str. 122.

${ }^{42}$ La Fontaine, Servante.

${ }^{43}$ B. Ćopić, Gluvi barut, Beograd - Sarajevo, 1975, str. 271. Cf. aussi: Laka roba - žena slabog morala. (RMS).
} 
$3^{\circ}$ Les formes sont semblables, mais le sens est différent (robe - roba). $4^{\circ} \mathrm{Ni}$ la forme ni le sens n'ont de points communs (coton - ćeten). Une analyse de l'évolution phonétique de ces paires de mots rend compte de leur forme actuelle dans les deux langues. Une autre analyse explique le développement du sens et les changements sémantiques en français et en serbe ainsi que dans les langues véhiculaires.

\section{REFERENCES BIBLIOGRAPHIQUES}

Livres

BELIĆ, Aleksandar. Osnovi istorije srpskohrvatskog jezika. Fonetika. Beograd, Naučna knjiga, 1976. IVŠIĆ, Stjepan. Slavenska poredbena gramatika. Zagreb, Školska knjiga, 1970. NiKOLIĆ, Svetozar. Staroslovenski jezik I. Beograd, Naučna knjiga, 1978.

POPOVIĆ, Mihailo. Istorija francuskog jezika. Beograd, Jasen, 2014.

\section{Dictionnaires}

DWB: GRIMM, J. \&W. DeutschesWörterbuch. 33 vol. Leipzig: S. Hirzel Verlag, 1854 - 1971. En ligne: http://woerterbuchnetz.de/cgi-bin/WBNetz/wbgui_py?sigle=DWB

FEW: Von Wartburg, Walter. 1922 - 1967. Französisches etymologisches Wörterbuch: eine Darstellung des galloromanischen Sprachschatzes, 25 vol. Basel: R. G. Zbinden. En ligne: https://apps.atilf.fr/lecteurFEW/

GODEFROY, Frédéric. 1881 - 1902. Dictionnaire de l'ancienne langue française et de tous ses dialectes du LXe au XVe siècle. 10 vol. Paris: F. Vieweg, Emile Bouillon. En ligne: http://micmap.org/dicfro/introduction/dictionnairegodefroy

KLAJN, Ivan et Milan ŠIPKA. Veliki rečnik stranih reči i izraza. Novi Sad, Prometej, 2006.

NișANYAN, Sevan. Sözlerin Soyağacı: Çăgdaș Türkçe Etimolojik Sözlü̆̆̈̈. Istanbul, AdamYay, 2007. En ligne: https://www.nisanyansozluk.com

PICOCHE, Juliette. Dictionnaire étymologique du français. Paris, Le Robert, 2009.

POKORNY, Julius. Indogermanisches etymologisches Wörterbuch. 3 vol. Berne: A. Francke, 1959. En ligne: https://archive.org/stream/indogermanisches01pokouoft\#page/n3/mode/2up

RMS: 1967 - 1976: Rečnik srpskohrvatskoga književnog jezika, Novi Sad - Zagreb, Matica srpska, Matica hrvatska.

REY, Alain. (sous la direction de), Dictionnaire historique de la langue française, 3 tomes, Paris, Le Robert, 1998.

SKOK, Petar.. Etimologijski rječnik hrvatskoga ili srpskoga jezika, 3 vol. Zagreb, JAZU, 1971 - 1973.

ŠKALJÍ, Abdulah. Turcizmi u srpskohrvatskom - hrvatskosrpskom jeziku. Sarajevo, Svjetlost, 1985.

TLF: Trésor de la Langue Française informatisé. Paris, CNRS édition, 2004.

VASMER, Max. Russisches Etymologisches Wörterbuch. Heidelberg: Winter, 1950 - 1958. En ligne: https://dic.academic.ru/contents.nsf/vasmer/

Vuk: KARADŽIĆ, Vuk. Srpski rječnik. Beograd, Štamparija Kraljevine Jugoslavije, 1935.

\section{NEKOLIKO FRANCUSKO-SRPSKIH ETIMOLOŠKIH PAROVA IZ LEKSIČKOG POLJA ODEVANJA}

$U$ članku se analizira fonetski i semantički razvoj nekoliko francuskih i srpskih reči koje pripadaju rečniku odevanja i koje imaju zajedničke etimone. To su reči istog indoevropskog ili latinskog $i$ reči arapskog $i$ turskog porekla. One se odnose na tekstilne materijale, tehniku izrade odeće $i$ na odevne predmete. Isptivanje promena oblika i značenja pokazuje da postoje četiri tipa odnosa između etimoloških parova. 1. Moguće je ustanoviti sličnost oblika i značenja u oba jezika (lin - lan, maillot - majica). 2. Postoji istovetnost značenja, ali se oblici veoma razlikuju (chanvre konoplja, coudre - šiti). 3. Sličnost oblika je očigledna, ali ne i sličnost značenja (robe - roba). 4. Ni značenje ni oblik nemaju zajedničkih odlika (coton - ćeten). Razlike u obliku i u značenju proizilaze iz evolutivnog procesa svojstvenog svakom jeziku, ali i usled uticaja jezika posrednika i analogije.

Ključne reči: etimologija, oblik, značenje, indoevropski, latinski, arapski 